\title{
Synthesis of Nuclei at the Limits of Nuclear Stability
}

\author{
P. Armbruster, GSI Darmstadt
}

The cosmos of isotopes is limited and the part accessible to man restricted. One of the tasks of nuclear physics is to explore the limits and constraints on the production and stability of hot nuclear systems and the essential requirements for a long-living ground state. At GSI, Darmstadt, for the first time the limit of instability to proton emission has been crossed and the limit of stability against spontaneous fission raised to the level of $Z=107$ and 109

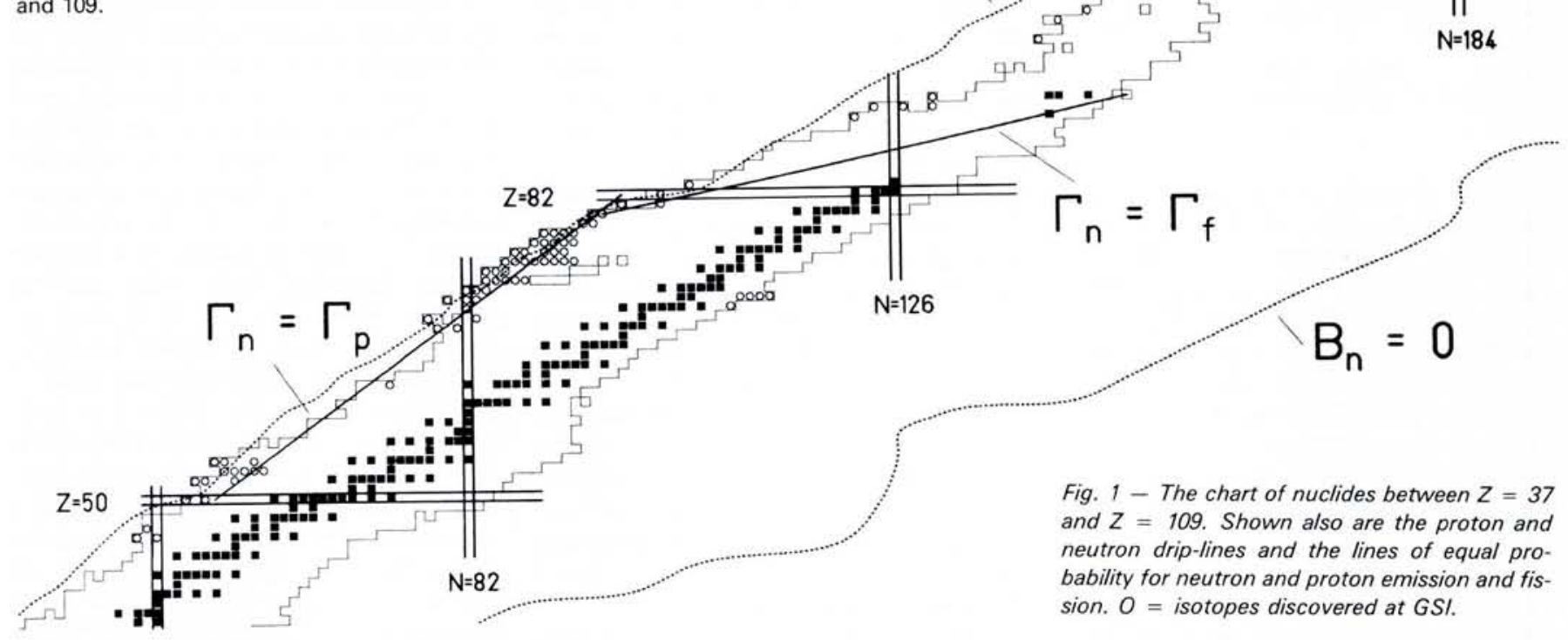

With heavy ion accelerators, nuclear reactions can be induced between all isotopes provided they are available for targets in mg-amounts or for beams in g-amounts. A large variety of new nuclear reactions have been found, but unfortunately these reactions in most cases lead to nuclei which have been known before. To produce new species, two reactions are of major interest: the fusion of two heavy nuclei and the massive transfer of nucleons observed in deep inelastic heavy ion reactions. If in the latter reaction one of the collision partners picks up predominantly neutrons, this mechanism may be used to produce neutron-rich new isotopes giving access to those regions on the neutron-rich side of the valley of stability which have not been explored by fission product studies. However, all the isotopes produced are far from the neutron drip-line, the line where nuclei become unstable to further increase in their neutron content.

Fusion reactions allow us to produce proton-rich isotopes, Fig. 1, as all fused systems possess less neutrons than the most stable isotopes at the corresponding atomic number. Isotopes at the proton drip-line become accessible up to the region of lead. Beyond the drip-line, protons are expected to tunnel through the Coulomb barrier in a new kind of radioactivity analogous to $\alpha$-decay. Moreover, the fused systems are heavier than each of the collision partners so that isotopes heavier than those existing naturally can be made. But, beyond lead, the disruptive Coulomb forces become more and more dominant, thus limiting the production of heavier and heavier elements.

Limitations on Fusion of Heavy Nuclei For the production of isotopes of elements beyond the natural ones, three conditions have to be fulfilled each of which may limit the feasibility of producing a given nucleus.

(1) To detect a nucleus, its half-life against spontaneous fission must be sufficiently long; ns-half-lives these days can be measured.

(2) The probability of fission during the cooling down phase of the fused compound system must be small enough to guarantee production rates of at least a few atoms per week.

(3) The Coulomb repulsion between the two collision partners during the fusion process must be small enough to allow the formation of a fused monosystem.

The disappearance of a barrier against spontaneous fission limits the detection of transiently formed isotopes near element 110 although nuclei with high fission barriers resulting from a stabilisation by shell effects at $N=184$ and $Z=114-126$ may give increased ground state stability to elements beyond $Z=114$. The isotopes of these superheavy elements are much more neutron rich than the heaviest nuclei made until now. The most stable nucleus ${ }^{298} 114$ cannot be reached by any combination of available isotopes, but as known regions of shell stabilisation are rather extended, there is hope that isotopes at accessible landing places still have high enough fission barriers to allow detection.

The losses during de-excitation of a fused compound system depend on the stability of nuclei against fission in states far above the ground state. Especially the temperature dependence of those contributions to the fission barrier which are due to shell effects are of importance. As all fusion reactions produce nuclei that are highly excited, the production of superheavy elements may be decided by such a dependence of shell effects on intrinsic excitation energy. To minimise the fission losses in the de-excitation phase, the highly fissionable nuclei should be produced with the smallest excitation energy possible. The colder the system formed, the better is its chances of survival. But how cold can fusion be?

Finally, the Coulomb repulsion between the nuclei may prevent the formation of a compound system. Passing the Coulomb barrier, an appreciable amount of the radial 


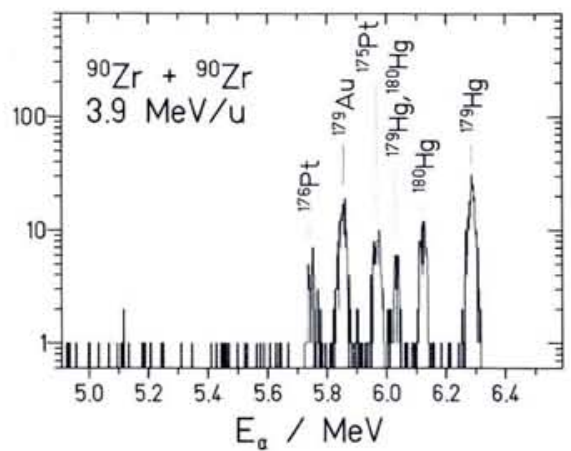

Fig. 2 - "Radiative fusion" is found in the reaction $90 \mathrm{Zr}+{ }^{90} \mathrm{Zr}-180 \mathrm{Hg}$ at $3.9 \mathrm{MeV} /$ nucleon. The $\alpha$-spectrum shows the $\gamma$-channel, 1n-channel, 1p-channel, and further daughter decays.

energy on the long way from a two-touching nuclei configuration to a compound system is transferred into intrinsic excitation energy. This energy has to be dissipated during the de-excitation phase, during which the losses by fission are increased. The energy transferred into excitation energy is reflected as a virtual increase of the Coulomb barrier, an "extra-push energy" is needed to fuse. This limitation in the entrance channel finally prevents the production of heavy elements, even if the deexcitation process could be survived and a ground state stability would exist.

Nuclear theory is able to calculate ground state properties of heavy nuclei. But to produce nuclei at the limits of stability an understanding of fusion dynamics and the behaviour of hot nuclei is needed as well. Progress here is of importance. Lacking other guidance, an interplay of reaction studies and production experiments was used to find ways to go beyond the elements reached previously. "Cold fusion" and "extra-push energy" are the new ingredients in the recipes of element making. They allowed the limit reached at $Z=$ 106 to be passed in the early seventies at Lawrence Berkeley Lab.

\section{Cold Fusion}

The excitation energy of a compound system is the difference between the energy to pass the Coulomb barrier and the reaction $\mathrm{Q}$-value. This value is smalier for the production of heavy compared to lighter nuclei. It is smaller given a certain mass of the fused system, if the latter is produced from symmetric collision partners than from more asymmetric ones. It is smaller, if especially stable nuclei are used as collision partners notably ${ }^{48} \mathrm{Ca},{ }^{90} \mathrm{Zr}$, ${ }^{136} \mathrm{Xe}$, and ${ }^{208} \mathrm{~Pb}$. It is smaller for fusion below the Coulomb barrier and it has been shown that there is fusion in addition to the quantummechanical barrier penetration. Some collective motions in nuclei are slower than the barrier passing time. The fluctuations in shape allow the two nuclei to fuse (with a small probability) at larger distances and hence a reduced Coulomb barrier. The phenomenon is shown to depend strongly on the nuclear structure of the collision partners.

The number of evaporated particles in the de-excitation cascade after fusion is a measure of the excitation energy. It has been decreased from the $4-5$ neutrons in the early fusion studies at Berkeley leading to elements $Z=101-106$, via 2 -neutron reactions found at Dubna, and 1-neutron de-excitation found in. 1979 at GSI, to a deexcitation by pure $\gamma$-emission found recently in the reaction ${ }^{90} \mathrm{Zr}+{ }^{90} \mathrm{Zr} \rightarrow{ }^{180} \mathrm{Hg}$ at GSI. The $\alpha$-spectrum of the isotopes produced in the reaction is presented in Fig. 2 . It shows below the barrier at an excitation energy of $17 \mathrm{MeV}$ the 1 proton-, 1 neutron-, and the $\gamma$-channel. The reaction combines all the advantages leading to a cold fusion reaction: heavy, symmetric, and shell stabilized collision partners fused below the barrier. The cross-section for "radiative fusion" is high, about $50 \mu \mathrm{b}$, as fission competition is still small. The fused system ${ }^{180} \mathrm{Hg}$ is situated at the triple point where proton and neutron separation energies and the fission barrier are equal and high, (Fig. 1). Around ${ }^{180} \mathrm{Hg}$ we find the nuclei the most resistive to intrinsic excitation. If these are produced cold, they reveal the long searched "radiative fusion", the ultimate in cold fusion.

\section{"Extra-Push"}

The ratio of disruptive Coulomb forces and attractive nuclear forces governs the amalgamation of two nuclei into one. For a monosystem this ratio is given by the fissility parameter $Z^{2} / A$. For a two-touching sphere configuration, the late R. Bass defined a corresponding parameter making use of the proximity force. Taking into ac-

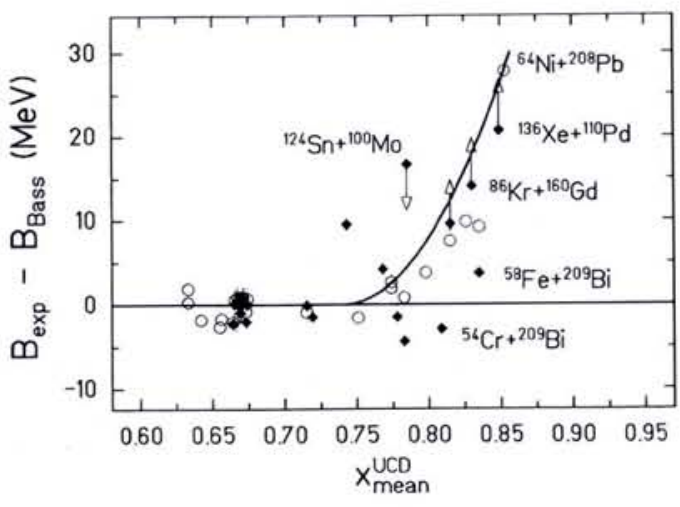

Fig. 3 - Evaporation residues ( $\$ ) and binary fragmentation $(\mathrm{O})$ experiments performed at GSI gave experimental evidence for the "extra-push" hypothesis. $\triangle B / M e V=2000\left(X_{\text {mean }}^{U C D}-0.74\right)^{2}$ gives the full line. count in addition that the proton and neutron ratio between the two partners is equilibrated very quickly (within times of $10^{-22} \mathrm{~s}$ ) and that the nuclear system at the Coulomb barrier for those systems where the "extra-push" becomes of importance, is more compact than the two-touching sphere configuration, a parameter $X_{\text {mean }}^{\cup C D}$ describing the ratio of Coulomb and nuclear forces has been defined, which is applied to organise the vast amount of data collected in the past years. Fig. 3 shows the extra-push energy using as a reference a Bass-potential to calculate the Coulomb barrier as a function of this parameter. The Coulomb forces surmounting $74 \%$ of the nuclear forces, the "extra-push" comes into play. It increases beyond this point

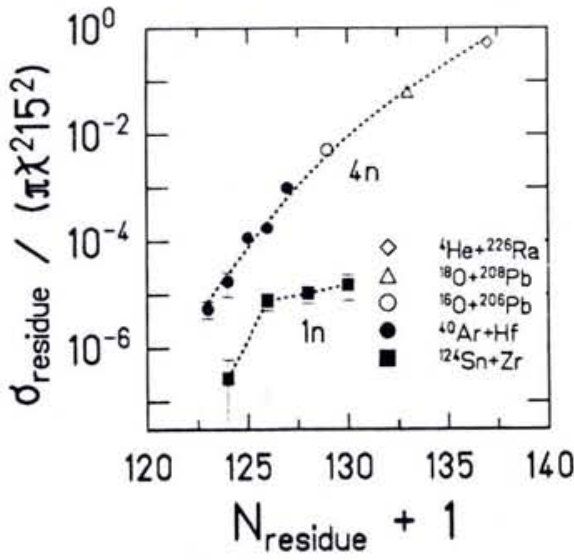

Fig. 4 - Evaporation residue cross section for the production of the "pseudo superheavies" around ${ }^{216} \mathrm{Th}$. The $4 n$-channel - $40 \mathrm{MeV}$ excitation energy - shows liquid drop behaviour and no preferential yield at $N=126$, whereas a slight increase is seen in the yield of the 1n-channel $20 \mathrm{MeV}$ excitation energy - indicating shell effects in spherical shell stabilized systems to become of importance in the production below this energy.

quadratically. Already at $84 \%$ about 20 $\mathrm{MeV}$ of additional energy is introduced into the system. The excitation energy at the barrier for reactions leading into the region of superheavy elements is for all possible combinations of isotopes increased by the extra-push heating. In total it amounts to at least $30 \mathrm{MeV}$.

\section{"The Pseudo-Superheavies"}

The crucial question at which temperature a spherical shell stabilised nucleus loses its stabilisation had to be elucidated in order to understand the prospects of superheavy element production. In any case beyond $Z=120$ no compound system with less than $50 \mathrm{MeV}$ intrinsic excitation energy can be produced. The study of shell stabilised, highly fissionable, spherical nuclei is possible making use of the $N=$ 126 shell closure. Nuclei around ${ }^{216} \mathrm{Th}$ are the nearest analogy to superheavy nuclei. They have been produced via fusion of different target-projectile combinations. The additional stability of these nuclei due to shell effects has been found to be obser- 
vable in the production cross-sections only at surprisingly small excitation energies below $20 \mathrm{MeV}$. At energies of about 30-40 $\mathrm{MeV}$ the minimum at which superheavy nuclei can be produced, the compound systems behave as if no shell stabilisation would exist, a discouraging finding in view of the chances to produce superheavy elements, Fig. 4. Extra-push heating and the liquid drop behaviour of spherical shell stabilised nuclei seen already at moderate excitation energies, may together act to prevent the production of superheavy nuclei by fusion.

Fig. 5 summarizes the finding of our fusion studies. In the triangle, Fig. 5(a), all reactions which have been successfully used to produce isotopes by fusion at GS are indicated as full points, open points indicate GSI- and other world wide used reactions, which have not led to any detected evaporation residues. The frontier between the two regions of points is given at an extra-push energy of about $10-15 \mathrm{MeV}$. Fig. 5 (b) shows the excitation energy at the barrier in the same presentation. Q-values, Coulomb-barriers, and extra-push energies go into this plot. The size of the squares stands for energies running from $10 \mathrm{MeV}$ to $50 \mathrm{MeV}$. The coldest compound systems are found for nearly symmetric collision partners leading to elements $Z=80-94$, and for systems using shell stabilised nuclei around lead as targets, leading to elements between 100 and 109. Here, using subbarrier fusion, excitation energies below 25

Fig. 5 - (a) The target-projectile combinations leading to evaporation residues $\boldsymbol{0}$, and no evaporation residues $O$. Between $X_{\text {mean }}^{U C D}=0.80$ and 0.84 the production goes down below the $100 \mathrm{pb}$ level.

(b) The excitation energy of the fused systems taking into account the extra-push energy. The squares run from 10-50 MeV. The "cold fusion" valleys at $Z_{T}=82$ and $Z_{p}=20$, and the "cold plane" near symmetry for $Z_{T}=42 \pm 6$ is seen.
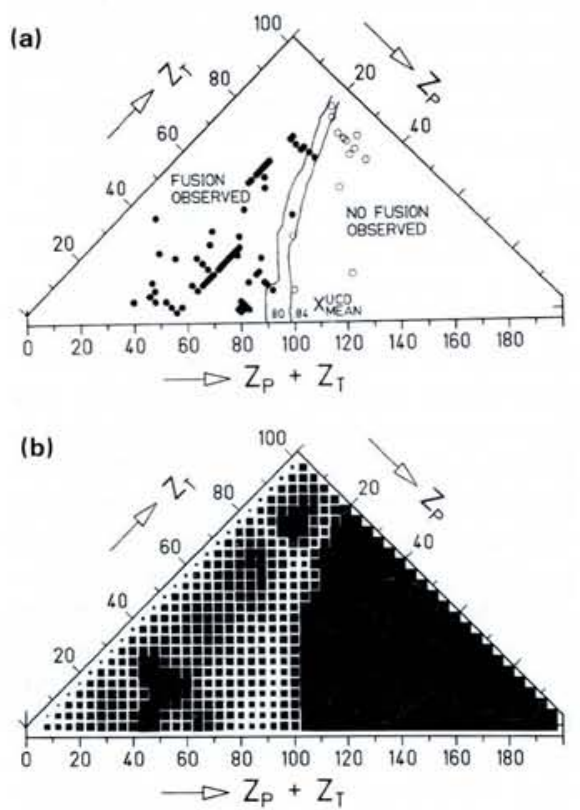

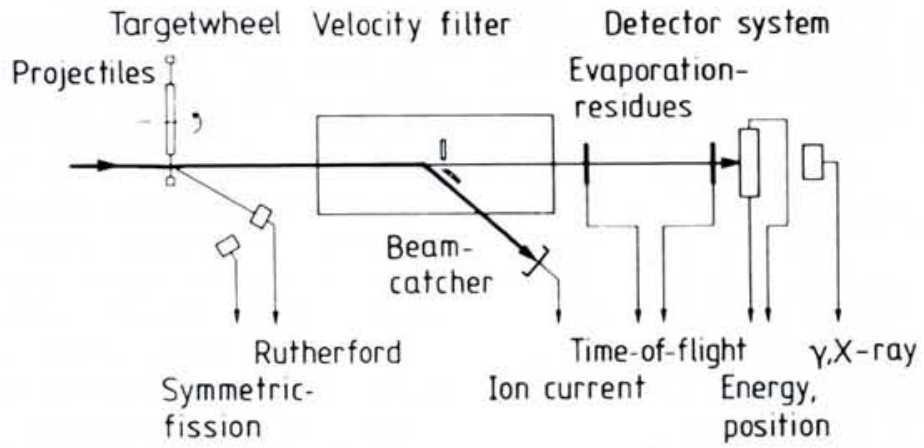

Fig. 6 - Fusion kinematics - velocity filter for recoils - recoil implantation - decay analysis made possible the construction of the highly efficient, fast separation device SHIP. The principal set-up used to detect single decaying atoms produced via fusion.

$\mathrm{MeV}$ have been reached. For heavier nuclei, the "extra-push" energy heats up the formed systems.

\section{The SHIP}

To search for rare isotopes, highly sensitive, universal, and rapid detection methods have to be made available. At GSI a new method has been developed which is adapted to the fusion reaction and which fulfils the above requirements more than any other previous technique, Fig. 6 . The momentum conservation requires for fusion that all systems travel in a small cone surrounding the beam direction with a velocity smaller than that of the projectiles inducing the reaction. The fused nuclei which are highly charged ions travelling with a few percent of the velocity of light are separated from the projectiles by a velocity filter - a combination of electric and magnetic fields which allows ions in a preselected velocity band to be separated out. This Separator for Heavy lon reaction Products - SHIP - enables the primary beam to be suppressed by factors of up to $10^{16}$ and generates a beam of all particles passing the filter in a given velocity window. This beam contains the fused systems, a background of degraded beam particles at a rate of about $100 / \mathrm{s}$, and eventually other reaction products which have the preset velocity. The separation time is the time-of-flight through the filter and amounts to about $1 \mu \mathrm{s}$. The separation is independent of the chemical properties of the fused systems, which leave SHIP with energies between $20 \mathrm{MeV}$ and $100 \mathrm{MeV}$. Efficiencies of $40 \%$ have been achieved.

Detection of the fused nuclei is through a system which makes full use of the fact that the energy obtained in the nuclear reaction has not been wasted. The velocity of all particles separated by SHIP is measured by a time-of-flight detector. In the focal plane, the energy, time of arrival, and position of each particle is registered by an array of position sensitive surface barrier detectors into which the particles are implanted. The radioactive decays of the implanted reaction products are registered by the same detector system. Energy, time of emission, and position from protons, $\alpha$-particles and fission products following the implantation are registered. Time correlations of events in the position windows defined by the implanted reaction product permit the decay chain to be reconstructed. The background in best cases is small enough still to correlate decays with halflives up to $30 \mathrm{~min}$. Events followed by decay chains can be detected unambigously allowing the identification of single decaying atoms, even if the production rates are of the order of one per week, a rate corresponding to a cross-section of $10^{-35} \mathrm{~cm}^{2}$.

\section{Proton Radioactivity}

Heavy ion accelerators have allowed for the first time, the extensive exploration of that region of nuclei, the de-excitation of which is dominated by proton emission. Fig. 1 shows as circles the ca. 90 isotopes produced at GSI using SHIP and an on-line separator facility. Most of them are in the region where prompt proton emission prevails. Protons are not only the main decay mode in the de-excitation cascade, they are found as delayed protons after $\beta$-decay, as well as protons from ground state decay. The latter process is the proton radioactivity searched for since 1915 - ground state to ground state decay by proton emission. It indicates that the proton drip-line has been crossed, and the proton is bound to the nucleus solely by a Coulomb well, out of which it is able to tunnel.

A systematic search for nuclei at the proton drip-line is of importance to nail down the binding energies of nuclei far from stability. Most of the new isotopes are $\alpha$-active, and from their $\alpha$-energies Q-values may be extracted, thus allowing the very proton rich nuclei to be directly connected via $\beta$-decay bridges to known masses. The prediction of the binding energies of nuclei like ${ }^{158} \mathrm{~W},{ }^{163} \mathrm{Os}$, and ${ }^{168} \mathrm{Pt}$ is one of the most stringent tests of a mass formula. The systematic investigation has enabled the candidates for proton radioactivity to be predicted. Above $N=84$ $\alpha$-decay is a competing fast decay mode. All search there failed. In the fusion of ${ }^{58} \mathrm{Ni}$ and ${ }^{96} \mathrm{Ru}$ via a $p, 2 n$-channel the isotope ${ }^{151} \mathrm{Lu}$ has been produced. It is protonradioactive. Fig. 7 shows the proton line found at $1.23 \mathrm{MeV}$; the half-life of the 
decay is $85 \mathrm{~ms}$. The $11 / 2$-ground state of ${ }^{151} \mathrm{Lu}$ decays via an emission of a $1.23 \mathrm{MeV}$ proton to the ground states of ${ }^{150} \mathrm{Yb}$. A second proton-emitter ${ }^{147} \mathrm{Tm}$ has been detected by the on-line-mass-separator technique. Search for further emitters is going on.

\section{Discovery of Elements 107 and 109}

In reactions between stable isotopes, all elements beyond $Z=100$ known so far can be made. The Dubna scientists were the first to leave the former technique of bombardment of actinide targets, which was successful up to $Z=106$. They showed in 1974 that fermium can be made via fusion of lead and argon. Bombarding ${ }^{208} \mathrm{~Pb}$ and ${ }^{209} \mathrm{Bi}$ targets with beams of ${ }^{40} \mathrm{Ar}$, ${ }^{48} \mathrm{Ca},{ }^{50} \mathrm{Ti},{ }^{54} \mathrm{Cr}$, and ${ }^{58} \mathrm{Fe}$ it was shown in Dubna and Darmstadt that all elements previously known can be made. We showed that the excitation energy of the formed systems is low, between $15 \mathrm{MeV}$ and 25 $\mathrm{MeV}$ and most of the isotopes are produced via $1 n$ - and $2 n$-channels. The fusion process is much colder than for actinide based reactions. The reactions gave a chance of producing the very fragile elements beyond $Z=106$, which have fission barriers between $1.5 \mathrm{MeV}$ and $2.5 \mathrm{MeV}$. Beyond $Z=$ 109 the extra-push heating in the $\mathrm{Pb}$-based combinations increases the excitation energy rapidly.

Fig. 8 shows decay chains of 262107 and 266 109. For $Z=107$, the real eka-rhenium searched for in the pre-fission age, seven events have been found in the $1 \mathrm{n}$-reaction ${ }^{54} \mathrm{Cr}+{ }^{209} \mathrm{Bi}$, for $Z=109$, one event has been seen in the reaction ${ }^{58} \mathrm{Fe}+{ }^{209} \mathrm{Bi}$. The isotopes made are odd-odd nuclei, which have a highly increased stability towards spontaneous fission. They are still $\alpha$-active, thus allowing the application of our chain analysis technique. The $\alpha$-energies of the two isotopes found are $10.37 \mathrm{MeV}$ and $11.10 \mathrm{MeV}$, their half-lives $5 \mathrm{~ms}$ and $3 \mathrm{~ms}$, their production cross-section $200 \mathrm{pb}$ and

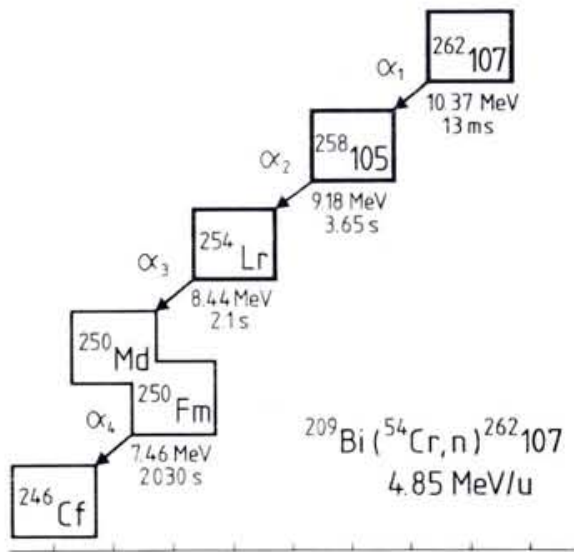

$\begin{array}{lllllllll}148 & 149 & 150 & 151 & 152 & 153 & 154 & 155 & 156\end{array}$

Neutron Number
$16 \mathrm{pb} .10^{11}$ nuclear encounters between ${ }^{58} \mathrm{Fe}$ and ${ }^{209} \mathrm{Bi}$ nuclei at a well-defined energy are necessary to produce one surviving nucleus of element 109. 109 is the element, where one detected event stands for all, remembering " $\varepsilon v$ to $\pi \alpha v$ " = "One for All" - a wisdom of Greek alchemists.

\section{Search for Superheavy Elements}

The most favourable reaction leading into the region of superheavy nuclei is ${ }^{48} \mathrm{Ca}$ on ${ }^{248} \mathrm{Cm}$ with an excitation energy at the Coulomb barrier of about $30 \mathrm{MeV}$. A collaboration with $\mathrm{LBL}$ made ${ }^{248} \mathrm{Cm}$-targets available at GSI. In a three week-search we looked for the production of isotopes of element 116. The measurement gave upper limits for the cross-sections of the order of $25 \mathrm{pb} / 10 \mathrm{MeV}$ within a half-life range from $10^{-6} \mathrm{~s}$ to $10^{6} \mathrm{~s}$. The final evaluation of the measurement is still in progress.

Why was the production of superheavy elements until now never successful? At a survival probability of 1 in $10^{11}$ out of all nuclear encounters, we are far from understanding why we make an element or we fail. There must be a limit where the production of heavier elements becomes unfeasible. To find this limit and to understand why it is, where it is, that is the question of relevance. Nature has given us an island of radioactive heavy elements from $Z$ $=84$ to $Z=109$. Most of the elements there have deformed nuclei. Elements beyond $Z=92$ have been produced with an enormous effort during the last decades. No-one has ever produced spherical nuclei beyond $Z=92$. Our reaction studies showed that only if produced cold, are their shell effects of importance. Superheavy nuclei may have ground state stability, but all recipes we applied produced them with high excitation energy as "extrapush" heating prevents a cold production.

(b)

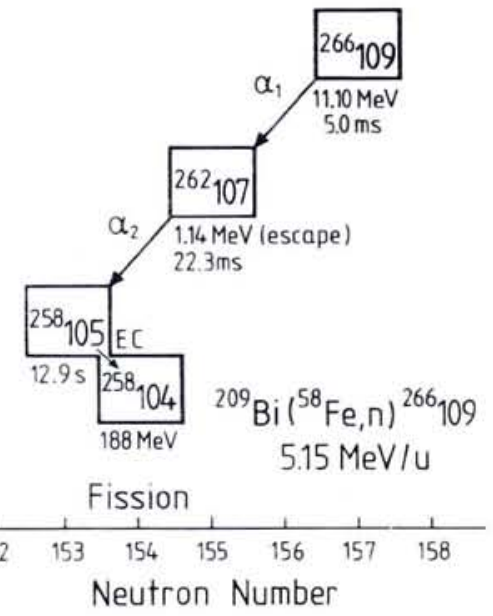

Fig. 8 - Decay chains observed in reactions of ${ }^{54} \mathrm{Cr}$ and ${ }^{58} \mathrm{Fe}$ with ${ }^{209} \mathrm{Bi}$ targets leading to two new elements.

(a) The decay of 262107, an isotope of the "ekarhenium" hunted in pre-fission age.

(b) The only atom of element 109 seen until now and its decay chain. It is attributed to the isotope 266 109. " $\mathrm{vV}$ to $\pi \alpha \mathrm{v}$ " in reference to the ancient wisdom.

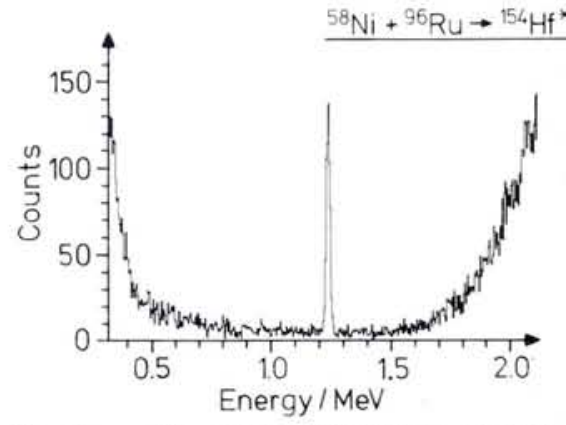

Fig. 7 - The proton-radioactivity of ${ }^{151} \mathrm{Lu}$ shown by its $1.23 \mathrm{MeV}$ proton line. This kind of radioactivity postulated in 1915 was discovered in 1981 at GSI.

Only a new fast mechanism, cooling the system before it fissions, may give us a chance to make them. Such a mechanism must be a non-statistical pre-equilibrium process, and may allow a gentle landing on the island of superheavy elements.

The described experiments have been carried out by a large group during 1976 -1983. The names of G. Münzenberg, S. Hofmann, W. Reisdorf, and K.-H. Schmidt stand for the about 25 scientists who helped to make the experiments a success. Collaboration with the University Giessen and Technische Hochschule Darmstadt was essential during all the years.

\section{University of Manchester}

\section{DEPARTMENT OF PHYSICS}

\section{Postdoctoral Research \\ Associate in Experimental Nuclear Structure Physics}

Applications are invited for the above post, funded by the SERC for the period 1 October 1983 until 31 January 1986 with the possibility of further extension.

The successful candidate will be expected to initiate and assist with research at the Nuclear Structure Facility, a 20/30 MV tandem Van de Graaff which is now operational at the Daresbury Laboratory. Exploitation of the on-line isotope separator at Daresbury Laboratory will also form a fraction of the candidate's duties.

Applicants are expected to hold a Ph.D. degree in Nuclear Structure Physics and should have an aptitude for pursuing research in experimental physics. Salary range $€ 7190-£ 8530$ p.a. (Superannuation).

Applications, with full C.V. and the names of two referees to:

Dr. R. Chapman,

Department of Physics,

The University,

Manchester M13 9PL

from whom further details may be obtained. 